\title{
Quantitative solutions for future energy systems and markets
}

\author{
Johann Hurink ${ }^{1}$ • Rüdiger Schultz ${ }^{2}$. \\ David Wozabal ${ }^{3}$
}

In the recent years, it has become more and more evident that anthropogenic climate change is real and happening at an accelerating pace, which is threatening modern society. The 2015 UN-climate conference in Paris brought about a global consensus that emission of climate active gases has to be drastically reduced to mitigate the effects of global warming.

Players in the energy sector, therefore, face rapidly changing conditions due to technological progress, the fast growth of renewables, market liberalization, and changes in global demand and supply. Firms, governments, and consumers are confronted with new challenges connected to increased competition, decentralization of production and the globalization of energy markets. These new problems call for tailored quantitative solutions to analyze, optimize, and control the operation of energy systems and markets, taking into account the distinct economic, technological and political characteristics of the energy sector.

Generally, energy systems are complex technical systems with strict real-time requirements and very high requirements in fault tolerance. Often these systems are embedded into markets that mirror the complexity of the technical system as, for example, is the case with power markets. The optimal design and operation of such

\footnotetext{
Johann Hurink

j.1.hurink@utwente.nl

Rüdiger Schultz

ruediger.schultz@uni-due.de

David Wozabal

david.wozabal@tum.de

1 University of Twente, Enschede, The Netherlands

2 Universität Duisburg-Essen, Essen, Germany

3 Technische Universität München, Munich, Germany
} 
systems and markets has to take into account technical, economic, as well as business perspectives. Problem arising in these areas are hard because they often involve multiple objectives and stakeholders, great uncertainty, and large systems that ignore political boundaries. Because of this complexity, stylized economic approaches as well as purely technical approaches fall short of the challenges that come with the design of a new energy system. Operations Research with its roots in optimization, statistics, and informatics can bridge this methodological gap and contribute to a new, sustainable energy system.

This special issue of OR-Spectrum contains a selection of papers that use the methods of Operations Research and have the potential to help to better understand the problems at hand and improve the quality of decisions.

\section{Tight MIP formulations of the power-based unit commitment problems}

Motivated by the infeasibilities in load coverage of power schedules representing staircase energy levels, Morales-España et al. (2015) provide the convex hull description for basic operations of slow- and quick-start units in power-based unit commitment. The model formulation distinguishes clearly between power and energy. The proposed constraints can be used to tighten mixed-integer programming formulations of unit commitment and bear potential of substantial improvement of computational performance.

A non-autonomous optimal control model of renewable energy production under the aspect of fluctuating supply and learning by doing

For renewable energy generation, it turns out to be difficult to guarantee a secure supply of energy as this supply underlies strong volatility. Furthermore, investment costs for these new technologies are so high that competitiveness with conventional energy forms is hard to achieve. To address this issue, Moser et al. (2016) analyze a non-autonomous optimal control model considering the optimal composition of a portfolio that consists of fossil and renewable energy and which is used to cover the energy demand of a small country.

Optimizing water tanks in water distribution systems by combining network reduction, mathematical optimization and hydraulic simulation

Decreased water consumption in Germany poses novel problems in maintaining efficient water production. Hallmann and Suhl (2016) propose a combination of network reduction, mathematical optimization, and hydraulic simulation to support planning of tanks in water distribution systems. A non-convex mixed-integer quadratically constrained optimization problem is solved approximately. Hydraulic differences due to model reduction and approximation are cleared by, if necessary multiple, iterations of model reduction and simulation.

\section{Valid inequalities for the topology optimization problem in gas network design}

Growing energy and gas demand in Europe and the requirement of reliable distribution make network extension a formidable task for which automatic planning tools supporting the decision process are extremely useful. Humpola et al. (2016) introduce a new class of valid inequalities tightening mixed-integer nonlinear programming relaxations of network expansion problems and speed up computations substantially this way. 


\section{Sell or store? An ADP approach to marketing renewable energy}

In deregulated markets, electricity is usually traded in advance, and the advance commitments have a time lag of several periods. Gönsch and Hassler (2016) consider this aspect for a producer that generates energy from stochastic, renewable sources, such as solar or wind and uses a storage device with conversion losses. They model the resulting commitment problem as a Markov Decision Process and solve it using an approximate dynamic programming approach. The presented approach is quite general with regard to the stochastic processes describing the energy production and price evolution.

\section{Perfect competition vs. strategic behaviour models to derive electricity prices and the} influence of renewables on market power

The exercise of market power on electricity markets has been a much discussed topic in many countries over the last years. Koschker and Möst (2016) test three of the most common concepts of competition with and without strategic interaction on the German electricity market. In particular, they try to explain observed prices with a simple model of perfect competition, a Cournot model, and a bilevel model. It turns out that, except for some high load hours, the perfect competition model performs best. Furthermore, the authors investigate how the ability to abuse market power varies with the amount of renewables in the system.

\section{The effect of intermittent renewables on the electricity price variance}

The hypothesis that production from renewables increases the price variance on electricity spot markets is reviewed critically by Wozabal et al. (2016). Identifying variance of infeed from intermittent sources (IES) and the shape of the industry supply curve as pivotal factors influencing the electricity price variance, they conclude from their model that the produced amount of IES is crucial, namely small to moderate quantities of IES tend to decrease whereas large quantities to increase the price of electricity.

\section{References}

Gönsch J, Hassler M (2016) Sell or store? An ADP approach to marketing renewable energy. doi:10.1007/ s00291-016-0439-x (this issue)

Hallmann C, Suhl L (2016) Optimizing water tanks in water distribution systems by combining network reduction. Math Optim Hydraul Simul. doi:10.1007/s00291-015-0403-1 (this issue)

Humpola J, Fügenschuh A, Koch T (2016) Valid inequalities for the topology optimization problem in gas network design. doi:10.1007/s00291-015-0390-2 (this issue)

Koschker S, Möst D (2016) Perfect competition vs. strategic behaviour models to derive electricity prices and the influence of renewables on market power. doi:10.1007/s00291-015-0415-x (this issue)

Morales-España G, Gentile C, Ramos A (2015) Tight MIP formulations of the power-based unit commitment problem. OR Spectr 37:929-950

Moser E, Grass D, Tragler G (2016) A non-autonomous optimal control model of renewable energy production under the aspect of fluctuating supply and learning by doing. doi:10.1007/s00291-016-0444-0 (this issue)

Wozabal D, Graf C, Hirschmann D (2016) The effect of intermittent renewables on the electricity price variance. doi:10.1007/s00291-015-0395-x (this issue) 